BIO Web of Conferences 2, 04001 (2014)

DOI: $10.1051 /$ bioconf/ 20140204001

C) Owned by the authors, published by EDP Sciences, 2014

\title{
Systems chemistry of $\alpha$-amino acids and peptides
}

\author{
Grégoire Danger $^{1}$, Laurent Boiteau ${ }^{2}$, Jean-Christophe Rossi ${ }^{2}$, and Robert Pascal ${ }^{2}$ \\ ${ }^{1}$ Spectrométries et Dynamique Moléculaire, Physique des Interactions Ioniques et Moléculaires, \\ UMR7345, CNRS - Aix-Marseille Université, Centre Saint-Jérôme - case 252, 13397 Marseille, \\ Cedex 20, France \\ ${ }^{2}$ Institut des Biomolécules Max Mousseron, UMR 5247 CNRS - Université Montpellier 1 \& \\ Montpellier 2, CC17006, Place E. Bataillon, 34095, Montpellier, France
}

\begin{abstract}
Pathways have been disclosed in the past decade, which support the possibility that $\alpha$-amino acids could have contributed to self-organization processes leading to the emergence of life. It is proposed that the systems chemistry of these simple building blocks may have led to features of self-organization through the realization of protometabolisms based on unidirectional loops involving both peptide formation and breakdown and additional feedback processes. Potential peptide activating agents have been identified. Scenarios of peptide elongation are proposed to account for peptide elongation both at the $\mathrm{N}$-terminus and the $\mathrm{C}$-terminus and new indications that these processes could be involved in symmetry breaking have been provided.
\end{abstract}

\section{Introduction}

Many observations and experiments lead to the conclusion that $\alpha$-amino acids can be formed abiotically in a diversity of environments in the Universe provided that oxygen is present at low levels. This is the case for instance in the interstellar medium where organic building blocks are formed and lead eventually to the presence of $\alpha$-amino acids in meteorites, which is now well recognized [1,2]. It was probably also the case in the atmosphere of the primitive Earth although in less abundant amounts than predicted by the Miller experiment $[3,4]$. Limited concentrations of $\alpha$ amino acids can also be formed from inorganic carbon and nitrogen in hydrothermal systems [5]. It is therefore likely that amino acids were present when life emerged. Though many researchers are involved in the study of a hypothetical RNA world [6] in which life could have emerged from nucleotide chemistry, it is likely that amino acids were at least early associated with processes involving nucleotides, which gives a first indication on how the translation process emerged [7]. This observation is strengthened by the recent report that the chemistries of amino acids and nucleotides precursors could be very closely associated [8]. However, having monomers constitutes only a part of the story and a contribution of $\alpha$-amino acids or even peptides to the origin and early developments of life needed additional processes likely to sustain improbable but dynamically stable states [9-15]. The new and improbable properties emerging this kind of processes are the object of study of Systems Chemistry [16,17]. We report here on the conceptual background leading our studies [18] and on the results of studies carried out to assess the possibilities of a potential Systems Chemistry of $\alpha$-amino acids and peptides [19-21]. 


\section{Protometabolisms of amino acids and peptides}

Non-equilibrium thermodynamics have established that the distance from equilibrium and the nonlinearity can drive a system towards self-organization [22]. As far as dynamically stable chemical systems are concerned, these two conditions mean $(i)$ that the nonequilibrium state must be permanently or repeatedly maintained by energy inputs and (ii) that feedback processes introduce nonlinearity. Replication processes [9-11] or autocatalysis [23] are then required to observe the corresponding behaviour. Except PNAs [24,25], peptides usually lack the base-pairing ability of nucleic acids. Within the scope of peptide chemistry autocatalysis is therefore more likely to induce nonlinearity than direct sequence replication. The emergence of network autocatalysis may result from reaction cycles in which further features of feedback are additionally present. A kind of peptide protometabolism can be devised if peptide formation competes with hydrolysis over geological time scales and if some sequences are capable to catalyse their own formation (Figure 1).

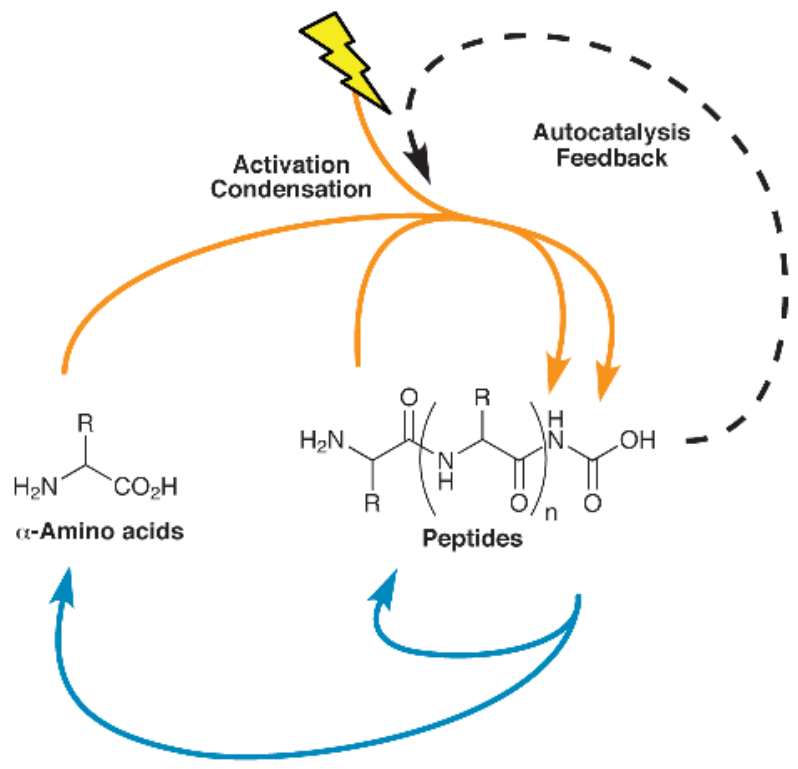

Hydrolysis

Fig. 1. Merging peptide formation and cleavage over geological time scales can lead to an amino acid and peptide protometabolism. The metabolic loop becomes unidirectional if peptide formation is coupled to a source of energy and can behave as a catalytic cycle. Positive feedback can transform the catalytic loop into an autocatalytic network.

Cyclic peptide protometabolisms have been proposed on the basis of activation processes leading to $\alpha$-amino acids $N$-carboxyanhydrides (NCAs), which constitute representative activated monomers capable of polymerizing [26,27]. Several potentially prebiotic pathways leading to NCAs have additionally been disclosed in the last decade [28-30] illustrating the plausibility of this pathway. A protometabolism of this kind has also been the starting point of the APED (ActivationPolymerization-Epimerizaton-Depolymerization) model [31] proposed for the emergence of homochirality (Figure 2). In this model, epimerization is proposed to occur at the N-terminal residue of a dipeptide introducing a possibility of reproduction of chirality when free amino acids are recovered by hydrolysis. The numeric analysis of this model supports the possibility of symmetry breaking in favourable cases corresponding to a wide domain of the set of rate constants of polymerization, epimerization and depolymerization reactions [31].

An experimental investigation of the practical feasibility of this model led to the conclusion that the ratio of diastereomers obtained after selective epimerization at the $\mathrm{N}$-terminal residue is not 
favourable to symmetry breaking [19]. Therefore, we have embarked on studies dedicated to assess an alternative possibility based on peptide elongation at the C-terminus.

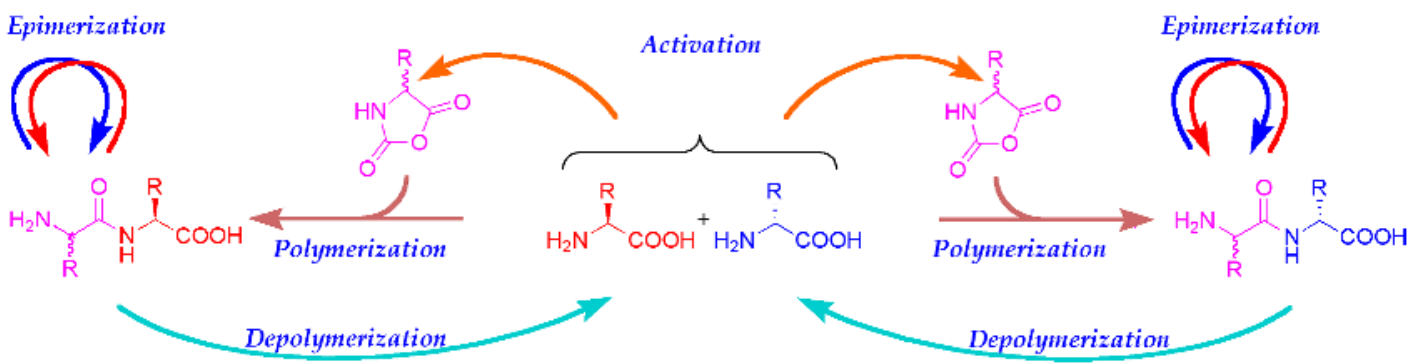

Fig. 2. Representation of the APED model as a competition between two autocatalytic loops. For certain sets of rate constants, one of the loops can become prevalent leading to spontaneous symmetry breaking.

\section{C-terminus activation in peptides}

\subsection{The need for activating agents}

Although the formation of peptides can take place under close to equilibrium conditions [5], a peptide protometabolism (Figure 1) associated with a dynamic steady state would require the process to proceed irreversibly $[7,12-15]$ and the cyclic arrangement to work unidirectionally. This observation means that the activating agent must contain both the free energy needed for the formation of the peptide bond and that required to ensure irreversibility, which strongly limits the set of species available in the environment to energy-rich species.

\subsection{Cyanate as an activating agent for carboxylic acids}

As cyanate was previously demonstrated as an activating agent capable of yielding $\alpha$-amino acid $N$-carboxyanhydrides (NCAs) from the free amino acids, we logically checked its activity in promoting carboxyl group activation. However, we observed that the degree of activation of cyanate is usually not sufficient to activate carboxylic acids, and no reaction occurred when attempting to couple acetyl alanine with glycine or glycine derivatives in water at $\mathrm{pH}$ values close to neutrality in the presence of cyanate [20]. But it was observed that succinic anhydride could be formed intramolecularly from succinic acid in the presence of cyanate. In the same way, peptides having a C-terminal aspartyl residue were observed to undergo coupling with cyanate as an activating agent (Figure 3) [20]. This possibility is enabled by the neighbouring group participation of the $\beta$-carboxyl group leading to a five-membered cyclic anhydride (Figure 3). 


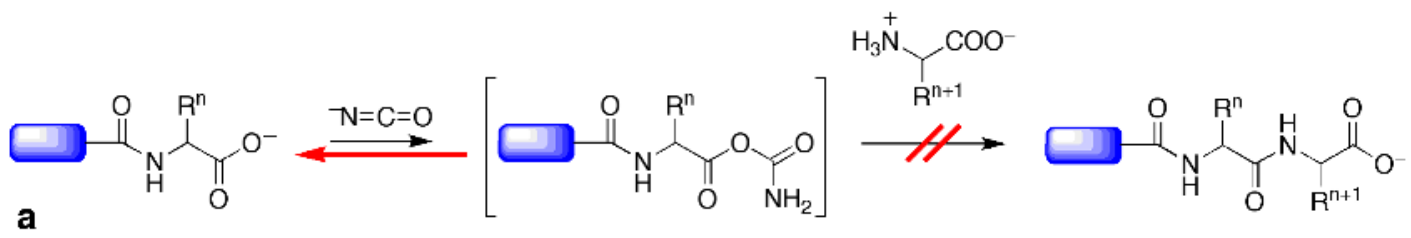<smiles>CC(C)CC(=O)NC(CC(=O)O)C(=O)NC(CC(=O)O)C(=O)OC(N)=O</smiles><smiles>CC=CCCC</smiles><smiles>CCCC(=O)NC(CC(=O)O)C(CC(=O)NCC1CCCC1)C(=O)[O-]</smiles>

b<smiles>COC1CC(NC(=O)C2CCCC2)C(=O)O1</smiles>

Fig. 3. Activation of carboxy terminus in peptides by cyanate. (a) Absence of reaction for residues without any reactive neighbouring group; (b) Specific behaviour of peptides with C-terminal aspartyl residues leading to reactive anhydride intermediates.

\subsection{Cyanamide}

Generalizing the intramolecular process to regular $\alpha$-amino acid residues imply the neighbouring peptide bond to be capable of nucleophilic attack on the activated $O$-acyl isourea adduct to undergo cyclization into a 5(4H)-oxazolone (Figure 4). Cyanamide was selected as a potential prebiotic reagent capable of performing this reaction since we found information about its conversion into carbodiimide [32,33].

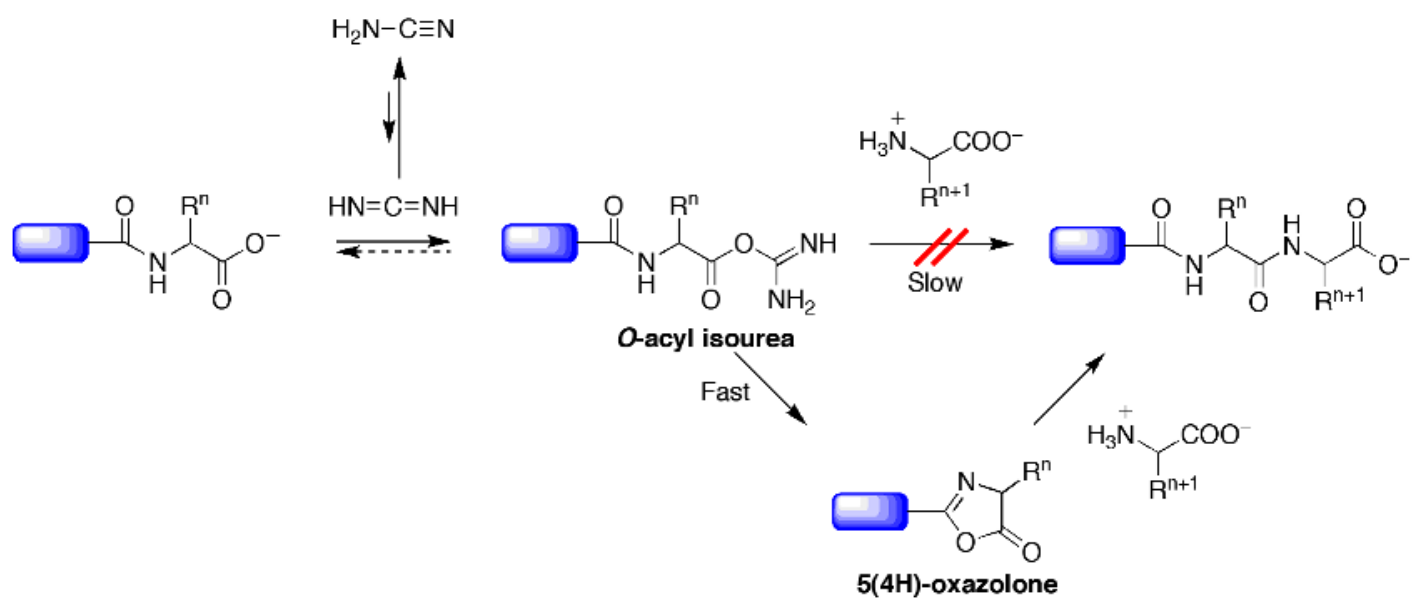

Fig. 4. Activation of carboxy terminus in peptides by cyanamide. The $O$-acylisourea intermediate can react intramolecularly to give the $5(4 H)$-oxazolones allowing further reactions with amino acids.

Although 5(4H)-oxazolone are well known to induce racemization in aqueous solution, their intermediacy improving peptide coupling in aqueous solution needed to be demonstrated. Information establishing this pathway have been obtained [21], which definitely shows that Cterminal elongation is a prebiotically relevant pathway [18]. The originality of this pathway lies in 
the fact that peptide coupling is associated with a high level epimerization at the C-terminal residue. This change of configuration at a selected position in the peptide suggests that a reproduction of chirality is possible at the same time as peptide elongation proceeds, which is worth of being studied more deeply because of its possible consequences on the emergence of homochirality that could take place in a way similar to the APED model [18], which would require a protometabolism of peptide based on C-terminus elongation to be established (Figure 5).

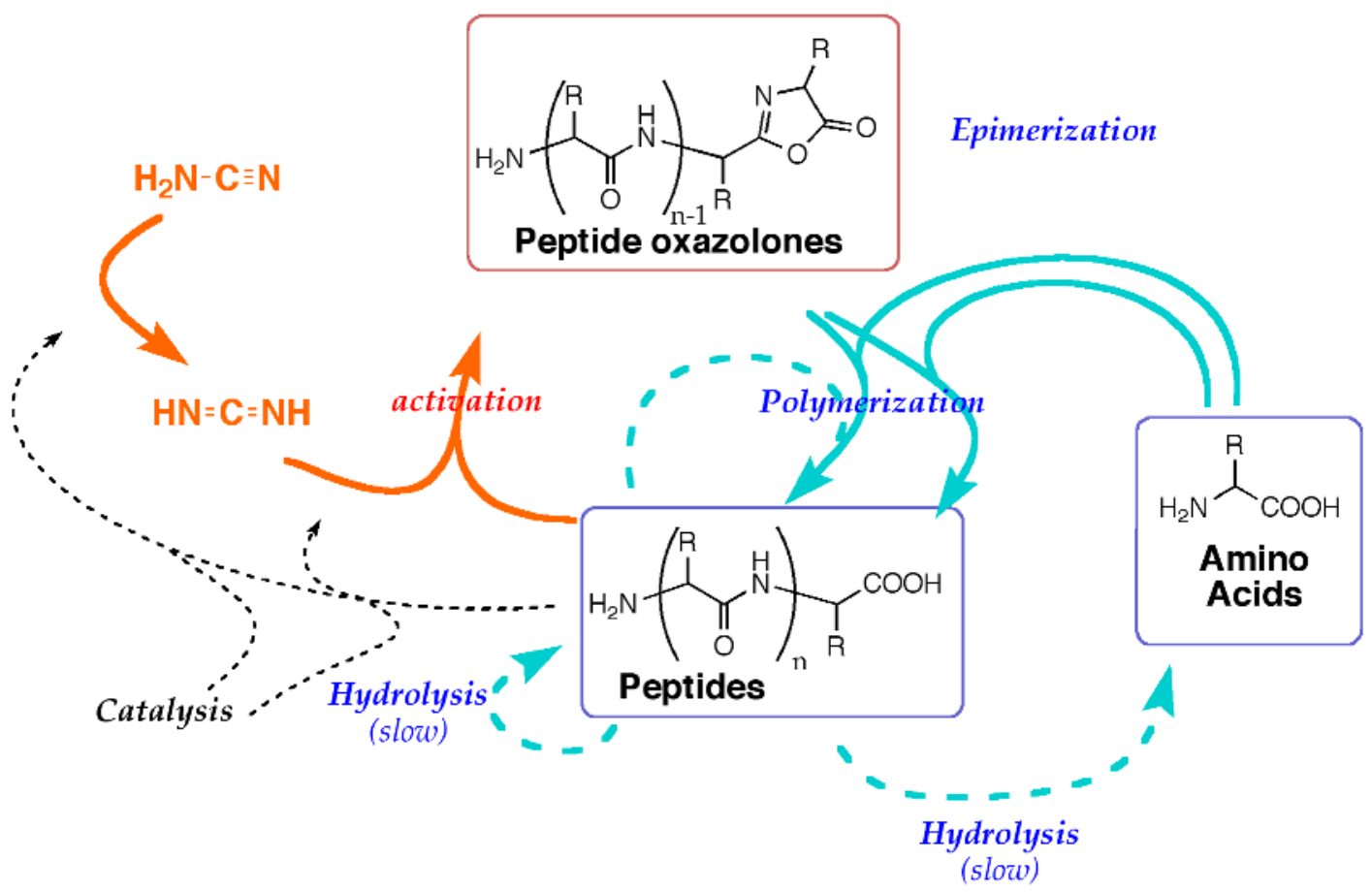

Fig. 5. A potential peptide protometabolism based on C-terminal elongation of peptides through activation by cyanate.

\subsection{Connection with RNA chemistry}

Peptide nucleic acids (PNAs) [25] have been proposed as early substitutes for nucleic acids on the grounds that the formation of $\alpha$-amino acids may be possible in a variety of environments, whereas that of nucleotides could only take place in trace amount and involves instable building blocks. Experimental support has been provided through the identification of pathways for the synthesis of diamino acid precursors of PNAs [35] and their identification in interstellar ice analogues [36]. However, a pathway leading to PNA monomers in prebiotic environments must involve the nucleic base moiety in addition to the amino acid backbone, the availability of a favoured process leading to the whole structure remains to be demonstrated. Moreover, this hypothesis is not supported by any biochemical indication that this kind of polymers may have played a role, and, though the hypothesis cannot be ruled out, it is difficult to historically support a path without any evidence [15]. Moreover, the formation of ribonucleotide monomers turned out to be less unlikely since it has been shown to take place directly from simple organic building blocks through a pathway by-passing the one derived from a biochemical disconnection [37-39]. The hypothesis that a coevolution of peptide and nucleic acid chemistries may have led directly to a protein and nucleic acid world in which translation had already evolved [40,30] is then a reasonable alternative to the RNA world [6]. The occurrence of activated intermediates in the chemistry of $\alpha$-amino acids and peptides is likely to 
have led to the formation of conjugates associating both kind of biomolecules. This has been demonstrated in the case of $\alpha$-amino acid $N$-carboxyanhydrides leading to mixed anhydrides reminiscent of aminoacyl adenylates by interaction with phosphates and nucleotides [41-43] (Figure 6). 5(4H)-Oxazolone are likely to give similar mixed anhydrides and the corresponding downhill less activated products with a large variety of chemical pathways that may be conceived [18].

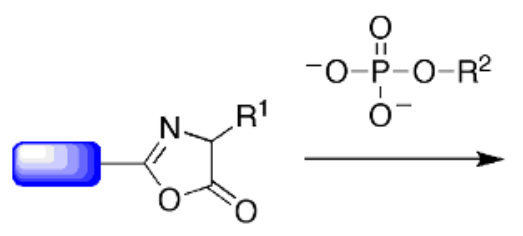

$5(4 H)$-oxazolone<smiles>[R]NC([R])C(=O)OP(=O)([O-])O[R]</smiles>

Mixed anhydrides

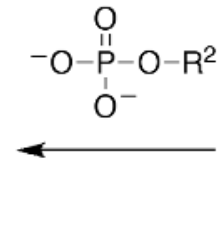

N-carboxyanhydride<smiles>[R]C1NC(=O)OC1=O</smiles>

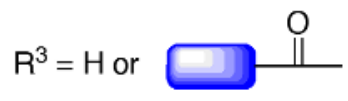

Fig. 6. The formation of mixed anhydrides observed from NCA and postulated from 5(4H)-oxazolones.

\section{Conclusion}

Prebiotic chemistry, defined as the chemistry taking place under conditions likely to promote selforganization towards life, is rich when considering amino acids and it can lead to multiple pathways. The formation of peptide is possible from free amino acids via the occurrence of activating agents enabling, in principle, unidirectional amino protometabolisms to sustain dynamically stable steady states that may have led to the emergence of features of self-organization and properties that could be reproduced. Coupled with a genetic polymer and especially through the ability of activated $\alpha$ amino acids to form mixed anhydrides with nucleotides, we can identify processes likely to lead to a world in which information could be stored and reproduced by base-pairing in sequences of nucleic acids whereas their function could be translated by the activity of peptides. Though we are far from being capable of providing a complete scenario, the emergence of specific properties taking advantage of their ability to be reproduced may not be implausible as for instance in the case of chirality, for which there is no need that a specific chemical species is reproduced, which could lead to selection and amplification in a way similar to natural selection. The analysis of the possibilities of $\alpha$-amino acid chemistry tend to confirm the view that chemical evolution and biological evolution can be considered as a single evolutionary process with no need of stating that a profound gap was separating them $[10,15]$.

\section{Acknowledgements}

The authors thank the interdisciplinary program Planetary Environments and Origins of Life (EPOV) supported by the CNRS and the CNES for funding this project and the COST Action CM0703 "Systems chemistry" for providing the possibility of fruitful scientific exchanges during the realization of this work. The authors are indebted to Solenne Charlot, Justine Canal, Manon Buchi, Arthur Michaut, and Damien Beaufils, Master and PhD students who have been involved in these studies and to the collaboration with Dr. Raphaël Plasson.

\section{References}

1. S. Pizzarello, E. Shock, Cold Spring Harb. Perspect. Biol. 2, a002105 (2010)

2. A. Burton, J. Stern, J. Elsila, D. Glavin, J. Dworkin, Chem. Soc. Rev. 41, 5459 (2012) 
3. S.L. Miller, Science 117, 528 (1953)

4. H.J. Cleaves, J.H. Chalmers, A. Lazcano, S.L. Miller, J.L. Bada, Orig. Life Evol. Biosph. 38, 105 (2008)

5. A.D. Aubrey, H.J. Cleaves, J.L. Bada, Orig. Life Evol. Biosph. 39, 109 (2009)

6. R.F. Gesteland, T.R. Cech, J.F. Atkins (eds), The RNA World, 2nd edn. (Cold SpringHarbor Laboratory Press, New York, 1999)

7. R. Pascal, L. Boiteau, Philos. Trans. R. Soc. Lond. B, Biol. Sci. 366, 2949 (2011)

8. D. Ritson, J. Sutherland, Nat. Chem. 4, 895 (2012)

9. A. Pross, Chem. Eur. J., 15, 8374 (2009)

10. A. Pross, J. Syst. Chem. 2, 1 (2011)

11. A. Pross, What is Life? How Chemistry Becomes Biology (Cambridge University Press, Cambridge, 2012)

12. L. Boiteau, R. Pascal Orig. Life Evol. Biosph. 41, 23 (2011)

13. R. Pascal, In I. W.M. Smith, C.S. Cockell, S. Leach (eds) Astrochemistry and Astrobiology: Physical Chemistry in Action (Springer, Berlin Heidelberg, pp. 243-269, 2012)

14. R. Pascal, J. Syst. Chem. 3, 3 (2012)

15. A. Pross, R. Pascal, Open Biol. 3, 120190 (2013)

16. G. von Kiedrowski, S. Otto, P. Herdewijn, J. Syst. Chem. 1, 1 (2010)

17. R.F. Ludlow, S. Otto, Chem. Soc. Rev. 37, 101 (2008)

18. G. Danger, R. Plasson, R. Pascal, Chem. Soc. Rev. 41, 5416 (2012)

19. G. Danger, R. Plasson, R. Pascal, Astrobiology, 10, 651 (2010)

20. G. Danger, S. Charlot, L. Boiteau, R. Pascal, Amino Acids 42, 2331 (2012)

21. G. Danger, A. Michaut, M. Bucchi, L. Boiteau, J. Canal, R. Plasson, R. Pascal, Angew. Chem. Int. Ed. 52, 611 (2013)

22. G. Nicolis, I. Prigogine, Self-organization in nonequilibrium systems. From dissipative structures to order through fluctuations (Wiley, New-York, 1977)

23. R. Plasson, A. Brandenburg, L. Jullien, H. Bersini, J. Phys. Chem. A 115, 8073 (2011)

24. P.E. Nielsen, M. Egholm, R.H. Berg, O. Buchardt, Science 254, 1497 (1991)

25. M. Egholm, O. Buchardt, L. Christensen, C. Behrens, S.M. Freier, D.A. Driver, R.H. Berg, S.K. Kim, B. Nordén, P.E. Nielsen, Nature 365, 566-568 (1993)

26. A. Commeyras, J. Taillades, H. Collet, L. Boiteau, O. Vandenabeele-Trambouze, R. Pascal, A. Rousset, L. Garrel, J.-C. Rossi, J.-P. Biron, et al., Orig. Life Evol. Biosph. 34, 35-55 (2004)

27. C. Huber, W. Eisenreich, S. Hecht, G. Wächtershäuser, Science, 301, 938 (2003)

28. L. Leman, L. Orgel, M.R. Ghadiri, Science 306, 283 (2004)

29. G. Danger, L. Boiteau, H. Cottet, R. Pascal, J. Am. Chem. Soc. 128, 7412 (2006)

30. R. Pascal, L. Boiteau, A. Commeyras, Top. Curr. Chem. 259, 69 (2005)

31. R. Plasson H. Bersini, A. Commeyras, Proc. Natl. Acad. Sci. U.S.A. 101, 16733 (2004) [DivPrebioPeptActiv19]

32. M.J. Sullivan, M.L. Kilpatrick, J. Am. Chem. Soc. 67, 1815 (1945)

33. M.L. Kilpatrick, J. Am. Chem. Soc. 69, 40 (1947)

34. F. Duvernay, T. Chiavassa, F. Borget, and J.-P. Aycard, J. Am. Chem. Soc. 126, 7772 (2004)

35. K.E. Nelson, M. Levy, S.L. Miller, Proc. Natl. Acad. Sci. U.S.A. 97, 3868 (2000)

36. C. Meinert, J.J. Filippi, P. de Marcellus, L. Le Sergeant d'Hendecourt, U.J. Meierhenrich ChemPlusChem 77, 186 (2012)

37. C. Anastasi, M.A. Crowe, M.W. Powner, J.D. Sutherland, Angew. Chem. Int. Ed. 45, 6176 (2006)

38. M.W. Powner, B. Gerland, J.D. Sutherland, Nature 459, 239-242 (2009)

39. M.W. Powner, J.D. Sutherland, J.W. Szostak, J. Am. Chem. Soc. 132, 16677 (2010)

40. V. Borsenberger, M.A. Crowe, J. Lehbauer, J. Raftery, M. Helliwell, K. Bhutia, T. Cox, J.D. Sutherland, Chem. Biodiversity 1, 203 (2004)

41. Biron, J.-P.; Pascal, R. J. Am. Chem. Soc. 126, 9198 (2004)

42. Biron, J.-P.; Parkes, A.L.; Pascal, R.; Sutherland, J.D. Angew. Chem. Int. Ed. 44, (2005)

43. L.J. Leman, L.E. Orgel, M.R. Ghadiri J. Am. Chem. Soc. 128, 20 (2006) 
